\title{
Two New Quinazoline Derivatives from the Moss Endophytic Fungus Aspergillus sp. and Their Anti-inflammatory Activity
}

\author{
Ning-Ning Wang ${ }^{1} \cdot$ Chun-Yu Liu ${ }^{1} \cdot$ Tian Wang $^{1} \cdot$ Yue-Lan $\mathrm{Li}^{1} \cdot \mathrm{Ke} \mathrm{Xu}^{1} \cdot$ Hong-Xiang Lou ${ }^{1}$
}

Received: 26 October 2020 / Accepted: 12 November 2020 / Published online: 21 November 2020

(c) The Author(s) 2020

\begin{abstract}
Two new quinazoline derivatives versicomides E (1) and F (2), and 10 known compounds (3-12) were isolated from the moss endophytic fungus Aspergillus sp. Their structures were determined on the basis of extensive spectroscopic data analysis and ECD calculations. Among them, the compound 7 (6-hydroxy-3-methoxyviridicatin) was first reported as a natural product. Inhibition on LPS-induced NO production in RAW 264.7 murine macrophages found that compounds $\mathbf{5 , 7}$ and $\mathbf{8}$ showed significant inhibitory effects on NO production, with $\mathrm{IC}_{50}$ values of $49.85,22.14$ and $46.02 \mu \mathrm{M}$ respectively.
\end{abstract}

\section{Graphic Abstract}
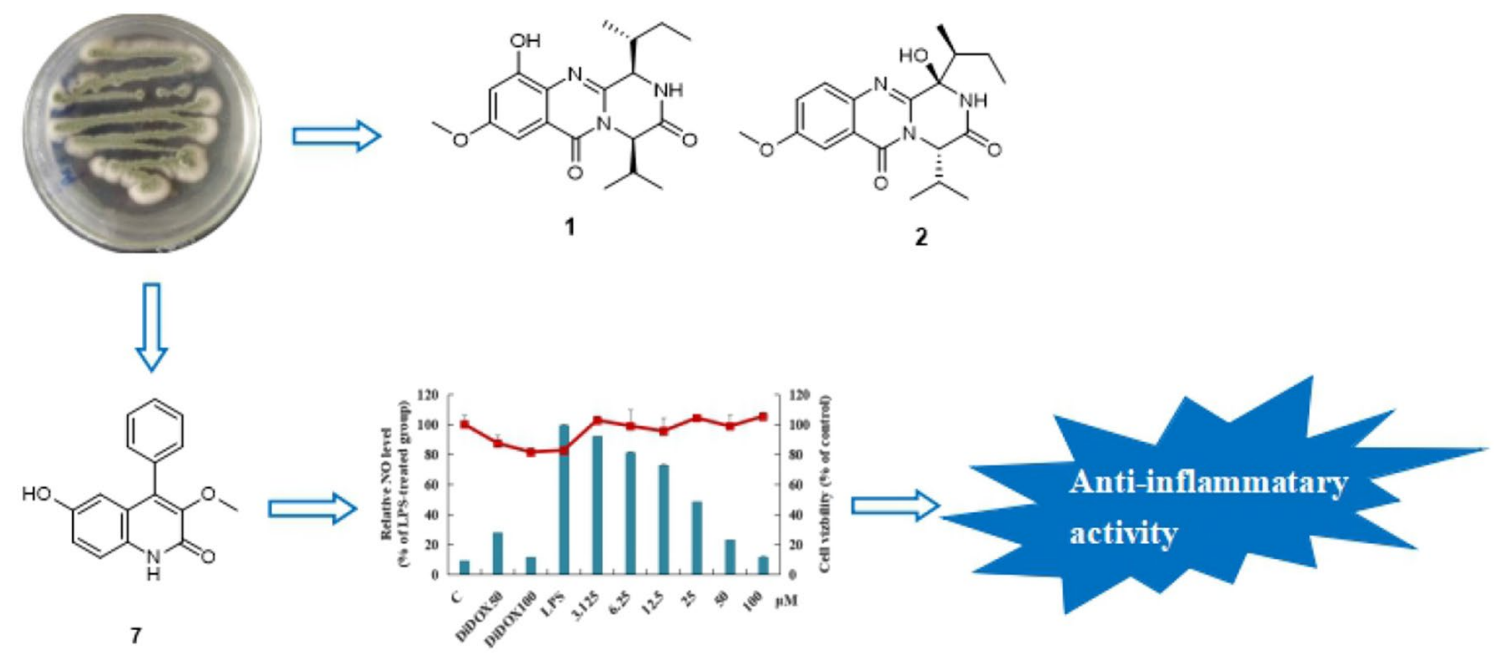

Keywords Endophytic fungus · Quinazoline · Anti-inflammatory activity

Ning-Ning Wang and Chun-Yu Liu have contributed equally to this work.

Electronic supplementary material The online version of this article (https://doi.org/10.1007/s13659-020-00287-5) contains supplementary material, which is available to authorized users.

Hong-Xiang Lou

louhongxiang@sdu.edu.cn

1 Department of Natural Product Chemistry, Key Lab of Chemical Biology of Ministry of Education, School of Pharmaceutical Sciences, Shandong University, Jinan 250012, China

\section{Introduction}

Endophytic fungi are defined as organisms that live in healthy internal tissues of plants without causing any immediate, obvious and negative effect [1,2], and have been considered as useful and peerless sources of molecules with potent bioactivity [3], such as cytotoxic [4], antibacterial [5], antifungal [6], antioxidative [7], neuraminidase inhibitory [8] and phosphodiesterase inhibitory activities [9]. The moss, as the oldest branch of terrestrial plant evolution, has built extensive contact with fungi [10]. The moss endophytic 
fungus is also a great resource for bioactive compounds, reports of which were not as many as other plant endophytic fungi. Previously, there were some compounds including alkaloids, cyclic peptide, anthraquinone with antifungal activity [11], cytotoxic activity [12, 13], immunosuppressive activity [14] and allelopathy activity [15] that were discovered in moss endophytic fungi. In the present research, two new quinazoline derivatives [16] versicomides $\mathrm{E}(\mathbf{1})$ and $\mathrm{F}(\mathbf{2})$ and 10 known compounds (3-12) were obtained from the moss endophytic fungus Aspergillus sp. It is also the first report of the presence of compound 7 (6-hydroxy-3-methoxyviridicatin) as a natural product. Furthermore, anti-inflammatory assay with lipopolysaccharide (LPS)-activated RAW 264.7 murine macrophages found that compounds 5, 7 and 8 showed strong inhibitory effects on NO production with $\mathrm{IC}_{50}$ values of $49.85,22.14$ and $46.02 \mu \mathrm{M}$ respectively.

\section{Results and Discussion}

Versicomide $\mathrm{E}(\mathbf{1})$ was isolated as faint yellow powder with the molecular formula $\mathrm{C}_{19} \mathrm{H}_{25} \mathrm{~N}_{3} \mathrm{O}_{4}$ by means of HRESIMS $\left(\mathrm{m} / \mathrm{z} 360.1919[\mathrm{M}+\mathrm{H}]^{+}\right.$, calcd. for 360.1923). The 1D NMR and HSQC spectra of compound $\mathbf{1}$ indicated that there was a methoxy $\left(\delta_{\mathrm{H}} 3.82, \delta_{\mathrm{C}} 55.5, \mathrm{CH}_{3}-22\right)$ and a $1,3,4,5$-tetrasubstituted benzene $\left(\delta_{\mathrm{C}} 131.1, \mathrm{C}-6 ; \delta_{\mathrm{C}} 154.1, \mathrm{C}-7 ; \delta_{\mathrm{H}} 6.83, \delta_{\mathrm{C}}\right.$ $107.7, \mathrm{C}-8 ; \delta_{\mathrm{C}} 58.6, \mathrm{C}-9 ; \delta_{\mathrm{H}} 6.99, \delta_{\mathrm{C}} 97.0, \mathrm{C}-10 ; \delta_{\mathrm{C}} 120.9$, $\mathrm{C}-11)$. Its ${ }^{13} \mathrm{C}$ NMR and HMBC spectra confirmed the existence of a Val moiety and a similar Ile moiety, which was also validated by COSY. Then, compared with signals of quinazoline derivatives [16], compound $\mathbf{1}$ had similar chemical shifts with versicomide A. The difference between them was that versicomide A had 1,3,4-trisubstituted benzene ring while $\mathbf{1}$ had 1,3,4,5-tetrasubstituted with and a hydroxyl group $\left(\mathrm{OH}-7, \delta_{\mathrm{H}} 9.80, \mathrm{~s}\right)$ as revealed by ${ }^{1} \mathrm{H}$ NMR. Therefore, the planar structure of $\mathbf{1}$ was elucidated as drawn in Fig. 1 which was also supported by the chemical shifts of C-7 $\left(\delta_{\mathrm{C}}\right.$ $154.1)$ and $\mathrm{C}-8\left(\delta_{\mathrm{C}} 107.7\right)$. The absolute configuration was confirmed by chemical calculation of the ECD spectrum. According to the literature [16], we presumed the absolute configuration was the same as versicomide A. However, by comparing the experimental ECD data to the calculated result, the experimental ECD was consistent with the calculated ECD of $(3 R, 14 R, 18 R)$ (Figs. 2, 3). Then, the absolute configuration of compound $\mathbf{1}$ was determined. The optical rotation of $[\alpha]_{\mathrm{D}}^{20}=-88.5(c 0.5, \mathrm{MeOH})$ also confirmed that.

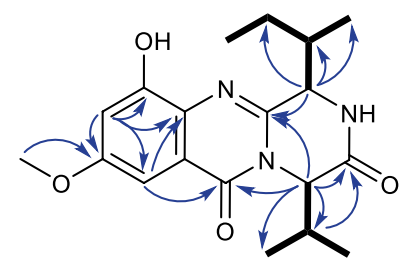

1

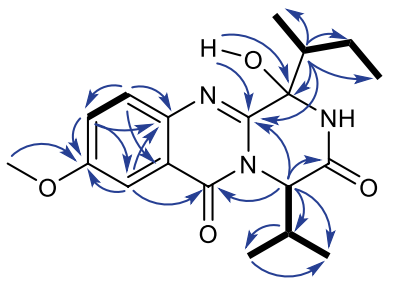

2
Fig. $2{ }^{1} \mathrm{H}-{ }^{1} \mathrm{H}$ COSY $(\mathrm{H}-\mathrm{H})$, key HMBC correlations $(\mathrm{H} \rightarrow \mathrm{C})$ of compounds $\mathbf{1}$ and $\mathbf{2}$<smiles></smiles>

1

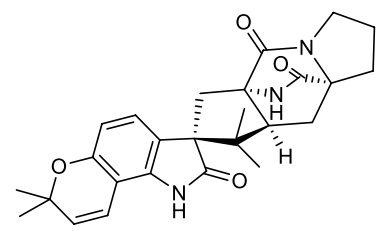

5

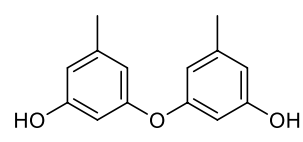

10<smiles>CCC(C)[C@]1(O)NC(=O)[C@@H](C(C)C)n2c1nc1ccc(OC)cc1c2=O</smiles>

2

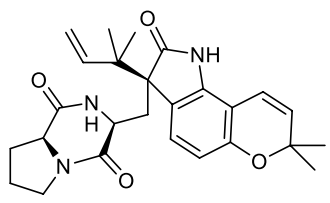

6

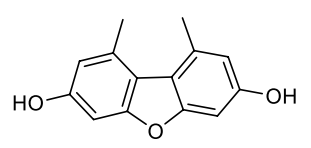

11

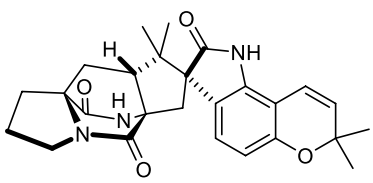

3<smiles>COc1c(-c2ccccc2)c2cc(O)ccc2[nH]c1=O</smiles>

7<smiles>Cc1cc(O)c2c(c1)Oc1c(C)cc(O)c(O)c1O2</smiles>

12

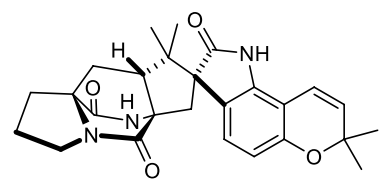

4<smiles>COc1c(-c2cccc(O)c2)c2ccccc2[nH]c1=O</smiles><smiles>CC(C)[C@H](C)/C=C/[C@H](C)[C@H]1CCC(=O)C23CC(=O)C4=CC(=O)CCC4(C)C2C[C@]13C</smiles>

8

9

Fig. 1 Structures of compounds 1-12 


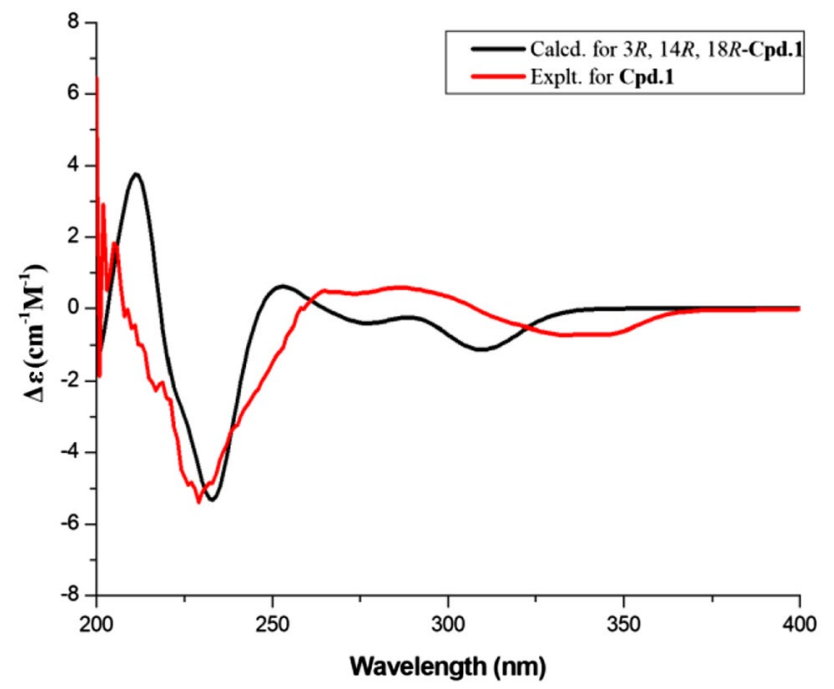

Fig. 3 Calculated and experimental ECD spectra for compound $\mathbf{1}$

Versicomide F (2) was also a faint yellow jelly. The molecular formula was determined to be $\mathrm{C}_{19} \mathrm{H}_{25} \mathrm{~N}_{3} \mathrm{O}_{4}$ by HRESIMS $\left(\mathrm{m} / \mathrm{z} 360.1919[\mathrm{M}+\mathrm{H}]^{+}\right.$, calcd. for 360.1923$)$. Its ${ }^{1} \mathrm{H}$ NMR showed that there was a different substitution from compound $\mathbf{1}$, and a different type of hydroxyl group $\left(\mathrm{OH}-3, \delta_{\mathrm{H}} 6.58, \mathrm{~s}\right)$ from $\mathrm{OH}-7\left(\delta_{\mathrm{H}} 9.80, \mathrm{~s}\right)$ in compound 1. Compound 2 had 1,3,4-trisubstituted benzene ring, lacking a hydroxyl substituent was determined for the correlations from $\mathrm{H}-7(7.64, \mathrm{~d}, 8.8 \mathrm{~Hz})$ to $\mathrm{C}-6, \mathrm{C}-8$, C-9 and C-11, together with the COSY correlation of H-7 and $\mathrm{H}-8$ in HMBC. HSQC indicated that C-3 $\left(\delta_{\mathrm{C}} 84.9\right)$ was an oxygenated quaternary carbon. Combination with the HMBC correlations from $\mathrm{OH}-3$ to $\mathrm{C}-3$ and C-4, the planar structure of compound $\mathbf{2}$ was depicted as in Fig. 1. By comparing the ECD of 2 (Fig. $S_{20}$ ) with that of the reported compound [16] leads to the final determination of the absolute configuration of compound 2 as $3 R, 14 S, 18 S$.

Compound 7 was reported as a natural product for the first time in this article. The structure of $\mathbf{7}$ was determined by comparing spectroscopic data with 3,6-O-dimethylviridicatin in literature [17] and determined as 6-hydroxy-3-methoxyviridicatin.

Other compounds were identified as versicolamide B (3) [18], taichunamide E (4) [19], notoamide B (5) [20], notoamide C (6) [21], 3-O-methylviridicatol (8) [17], dankasterone B (9) [22], diorcinol (10) [23], 3,7-dihydroxy-1,9-dimethyldibenzofuran (11) [24], aspergilol E (12) [25] by direct comparison of their spectral data with the reported.

All compounds were evaluated for the anti-inflammatory activity with the model to inhibit NO production in LPS-stimulated RAW 264.7 murine macrophages. Compounds $\mathbf{5 , 7}$ and $\mathbf{8}$ displayed strong inhibitory effects on
NO production, with $\mathrm{IC}_{50}$ values of $49.85,22.14$ and $46.02 \mu \mathrm{M}$ (Fig. 4) respectively.

\section{Experimental}

\subsection{General Experimental Procedures}

Optical rotations were acquired using a PerkinElmer 241MC polarimeter (Anton Paar GmbH, Graz, Austria) at $20{ }^{\circ} \mathrm{C}$. UV data were obtained by a UV-2450 spectrophotometer (Shimadzu, Japan). CD spectra were performed on a Chirascan spectropolarimeter (Applied Photophysics Ltd., Leatherhead, UK). NMR spectra were recorded on a Bruker Avance spectrometer operating at $400\left({ }^{1} \mathrm{H}\right)$ and $100\left({ }^{13} \mathrm{C}\right) \mathrm{MHz}$ or at $600\left({ }^{1} \mathrm{H}\right)$ and $150\left({ }^{13} \mathrm{C}\right) \mathrm{MHz}$ with tetramethylsilane as an internal standard. HRESIMS data were determined by using a Finnigan LC-QDECA mass spectrometer. IR spectra were recorded on a Nicolet iN 10 Micro FTIR spectrometer. HPLC were performed on an Agilent 1200 G1311A pump equipped with a G1322A degasser, a G1315D DAD detector, and an Eclipse XDB$\mathrm{C}_{18} 5 \mu \mathrm{m}$ column $(9.4 \times 250 \mathrm{~mm})$. Column chromatography (CC) was carried out using silica gel (200-300 mesh; Qingdao Haiyang Chemical Co. Ltd., Qingdao, China) and Sephadex LH-20 (25-100 mm; Pharmacia Biotek, Denmark). TLC was carried out with precoated silica gel GF-254 glass plates (Qingdao Haiyang Chemical Co. Ltd., Qingdao, China). The compounds were visualized under UV (254 nm) light and by spraying with anisaldehyde- $\mathrm{H}_{2} \mathrm{SO}_{4}$ followed by heating.

\subsection{Fungal Material}

The fungus Aspergillus sp. was isolated from the Trichocoleaceae sp., collected from Wuyanling Nature Reserve Area, Zhejiang Province, China. The strain (No. 7-2-1) was identified using nuclear ITS rDNA sequences (GenBank MN310533.1), and was deposited in the Key Lab of Chemical Biology of Ministry of Education, Shandong University, Jinan, China.

The fungus was cultured in eight $300 \mathrm{~mL}$ Erlenmeyer flasks, each containing $100 \mathrm{~mL}$ of potato dextrose broth (PDB), and put them on a rotary shaker $(120 \mathrm{rpm})$ at $25^{\circ} \mathrm{C}$ for 7 days. Large-scale fermentation was conducted in 60 Erlenmeyer flasks $(500 \mathrm{~mL})$, containing $80 \mathrm{~g}$ of rice and $120 \mathrm{~mL}$ of distilled $\mathrm{H}_{2} \mathrm{O}$. Then autoclave at $120{ }^{\circ} \mathrm{C}$ for $30 \mathrm{~min}$, and after cooling to room temperature, each flask was inoculated with $10 \mathrm{~mL}$ seed culture and incubated under static condition at room temperature for 50 days. 
5

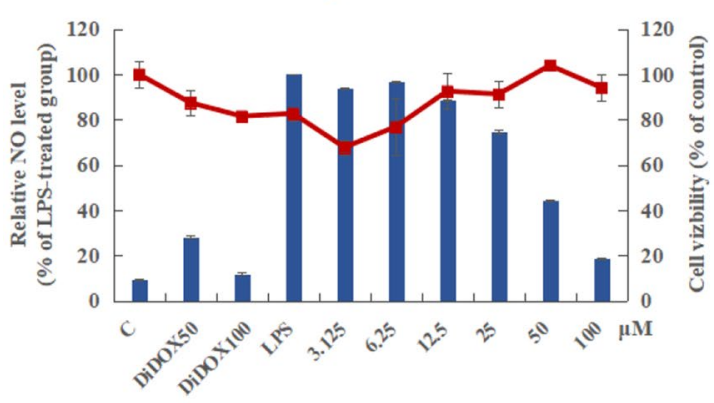

8

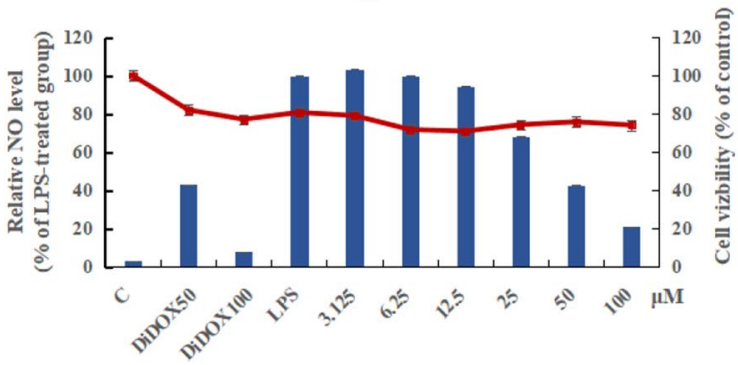

Fig. 4 Inhibitory activity of compounds $\mathbf{5 ,} 7$ and $\mathbf{8}$ against NO production in RAW 264.7 cells. Cells were treated with various concentrations of compounds along with LPS $(1 \mu \mathrm{g} / \mathrm{mL})$ for $24 \mathrm{~h}$, and the accumulation of nitrite was evaluated by Griess reagent. Values were

\subsection{Extraction and Isolation}

The fermented material including the mycelium was extracted with EtOAc for three times, and the organic solvent was evaporated under vacuum to yield the crude extract $(108 \mathrm{~g})$. The crude extract was separated into 15 fractions (A-O) using a silica gel column with a step gradient of $\mathrm{CH}_{2} \mathrm{Cl}_{2} / \mathrm{MeOH}$ from 100:0 to 0:100 (v/v).

Fr. D (4.9 g) was chromatographed by a silica gel column with step gradient of PE/EtOAc from 100:0 to 0:100 (v/v) to yield 15 subfractions $\left(\mathrm{D}_{\mathrm{A}}-\mathrm{D}_{\mathrm{N}}\right)$. Fr. $\mathrm{D}_{\mathrm{M}}(16.2 \mathrm{mg})$ was purified by HPLC $\left(80 \% \mathrm{MeOH} / \mathrm{H}_{2} \mathrm{O}, 1.5 \mathrm{~mL}\right)$ to yield compound $6\left(2.4 \mathrm{mg}, t_{\mathrm{R}}=14.0 \mathrm{~min}\right)$. Fr. $\mathrm{D}_{\mathrm{N}}(304.7 \mathrm{mg})$ and Fr. $D_{\mathrm{H}}(810.0 \mathrm{mg})$ were both isolated with Sephadex LH-20 $\mathrm{CC}$ eluted with $\mathrm{CH}_{2} \mathrm{Cl}_{2} / \mathrm{MeOH}(1: 2$ and 1:1) to afford 8 parts $\left(\mathrm{D}_{\mathrm{N} 1}-\mathrm{D}_{\mathrm{N} 8}\right.$ and $\left.\mathrm{D}_{\mathrm{H} 1}-\mathrm{D}_{\mathrm{H} 8}\right)$. Fr. $\mathrm{D}_{\mathrm{N} 3}(14.4 \mathrm{mg})$ was prepared with $\mathrm{HPLC}\left(72 \% \mathrm{MeOH} / \mathrm{H}_{2} \mathrm{O}, 1.5 \mathrm{~mL}\right)$ to yield compound $10\left(1.0 \mathrm{mg}, t_{\mathrm{R}}=13.0 \mathrm{~min}\right)$. Fr. $\mathrm{D}_{\mathrm{H} 2}(385 \mathrm{mg})$ was separated into 17 parts $\left(\mathrm{D}_{\mathrm{H} 2 \mathrm{a}}-\mathrm{D}_{\mathrm{H} 2 \mathrm{q}}\right)$ using MPLC (ODS, MeOH/ $\mathrm{H}_{2} \mathrm{O}$ from 10:90 to 100:0). Further purification of Fr. $\mathrm{D}_{\mathrm{H} 2 \mathrm{f}}$ $(15.0 \mathrm{mg})$ and Fr. $\mathrm{D}_{\mathrm{H} 2 \mathrm{~m}}(18.3 \mathrm{mg})$ with HPLC gave compound $1\left(40 \% \mathrm{MeCN} / \mathrm{H}_{2} \mathrm{O}, 1.5 \mathrm{~mL}, 2.8 \mathrm{mg}, t_{\mathrm{R}}=12.2 \mathrm{~min}\right)$, $2\left(40 \% \mathrm{MeCN} / \mathrm{H}_{2} \mathrm{O}, 1.5 \mathrm{~mL}, 6.2 \mathrm{mg}, t_{\mathrm{R}}=10.0 \mathrm{~min}\right)$ and 9 $\left(80 \% \mathrm{MeCN} / \mathrm{H}_{2} \mathrm{O}, 1.5 \mathrm{~mL}, 6.4 \mathrm{mg}, t_{\mathrm{R}}=14.0 \mathrm{~min}\right)$. Compound $11\left(2.7 \mathrm{mg}, t_{\mathrm{R}}=20.5 \mathrm{~min}\right)$ was obtained by purifying Fr. $\mathrm{D}_{\mathrm{H} 7}(60.7 \mathrm{mg})$ with $\mathrm{HPLC}\left(62 \% \mathrm{MeOH} / \mathrm{H}_{2} \mathrm{O}, 1.5 \mathrm{~mL}\right)$.
7

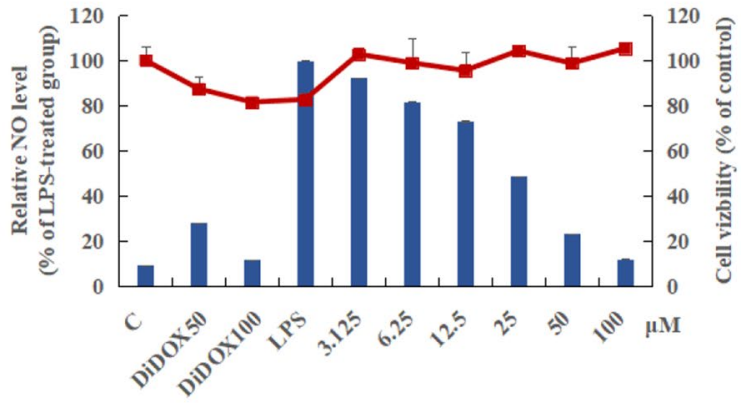

presented as mean \pm SD from three independent experiments. Column relative NO level, Dot cell viability, $C$ control, $D i D O X$ positive control

Fr. G (8.7 g) was chromatographed by a silica gel column with step gradient of PE/EtOAc from 100:0 to 0:100 (v/v) to yield 23 subfractions $\left(\mathrm{G}_{\mathrm{A}}-\mathrm{G}_{\mathrm{w}}\right)$. Fr. $\mathrm{G}_{\mathrm{M}}$ $(3.0 \mathrm{~g})$ was first fractionated by Sephadex LH-20 CC eluted with $\mathrm{CH}_{2} \mathrm{Cl}_{2} \mathrm{MeOH}$ (1:1) into seven subfractions $\left(\mathrm{G}_{\mathrm{M} 1}-\mathrm{G}_{\mathrm{M} 7}\right)$. Fr. $\mathrm{G}_{\mathrm{M} 5}(3.0 \mathrm{~g})$ was isolated into twelve parts $\left(\mathrm{G}_{\mathrm{M} 5 \mathrm{a}}-\mathrm{G}_{\mathrm{M} 51}\right)$ by MPLC (ODS, $\mathrm{MeOH} / \mathrm{H}_{2} \mathrm{O}$ from 10:90 to $100: 0)$, and then, Fr. $\mathrm{G}_{\mathrm{M} 5 \mathrm{j}}(69.9 \mathrm{mg})$ was subjected to Sephadex LH-20 CC by elution with $\mathrm{MeOH}$ to obtain six parts $\left(\mathrm{G}_{\mathrm{M} 5 \mathrm{j} 1}-\mathrm{G}_{\mathrm{M} 5 \mathrm{j} 6}\right)$. Preparation of Fr. $\mathrm{G}_{\mathrm{M} 5 \mathrm{j} 5}(49.5 \mathrm{mg})$ with HPLC $\left(77 \% \mathrm{MeOH} / \mathrm{H}_{2} \mathrm{O}, 1.5 \mathrm{~mL}\right)$ gave compound $12\left(2.0 \mathrm{mg}, t_{\mathrm{R}}=4.2 \mathrm{~min}\right)$.

Fr. I $(553.5 \mathrm{mg})$ was first fractionated using Sephadex $\mathrm{LH}-20 \mathrm{CC}$ with the mobile phase of $\mathrm{CH}_{2} \mathrm{Cl}_{2} / \mathrm{MeOH}$ (2:1), and 11 subfractions $\left(\mathrm{I}_{1}-\mathrm{I}_{11}\right)$ were obtained. Fr. $\mathrm{I}_{2}$ $(69.0 \mathrm{mg})$ was purified by $\mathrm{HPLC}\left(45 \% \mathrm{MeCN} / \mathrm{H}_{2} \mathrm{O}\right.$, $1.5 \mathrm{~mL})$ to afford compound $3\left(15.8 \mathrm{mg}, t_{\mathrm{R}}=15.0 \mathrm{~min}\right)$, $4\left(5.7 \mathrm{mg}, t_{\mathrm{R}}=16.0 \mathrm{~min}\right)$, and $5\left(3.9 \mathrm{mg}, t_{\mathrm{R}}=20.0 \mathrm{~min}\right)$. Fr. $\mathrm{I}_{5}(53.8 \mathrm{mg})$ was isolated using Sephadex LH-20 CC eluted with $\mathrm{MeOH}$, and got 7 subfractions $\left(\mathrm{I}_{51}-\mathrm{I}_{57}\right)$. Purification of Fr. $\mathrm{I}_{53}(42.7 \mathrm{mg})$ by HPLC $\left(54 \% \mathrm{MeOH} / \mathrm{H}_{2} \mathrm{O}\right.$, $1.5 \mathrm{~mL})$ gave compound $7\left(2.4 \mathrm{mg}, t_{\mathrm{R}}=17.0 \mathrm{~min}\right)$ and $\mathbf{8}$ $\left(2.6 \mathrm{mg}, t_{\mathrm{R}}=23.8 \mathrm{~min}\right)$. 
Table $1{ }^{1} \mathrm{H}$ and ${ }^{13} \mathrm{C}$ NMR spectral data for compounds $\mathbf{1}$ and $\mathbf{2}$ in DMSO- $d_{6}$

\begin{tabular}{|c|c|c|c|c|}
\hline \multirow[t]{2}{*}{ Position } & \multicolumn{2}{|l|}{1} & \multicolumn{2}{|l|}{2} \\
\hline & $\delta_{\mathrm{H}}{ }^{\mathrm{a}}(\mathrm{J}$ in $\mathrm{Hz})$ & $\delta_{\mathrm{C}}^{\mathrm{b}}$ & $\overline{\delta_{\mathrm{H}}{ }^{\mathrm{c}}(J \text { in } \mathrm{Hz})}$ & $\delta_{\mathrm{C}}^{\mathrm{d}}$ \\
\hline 1 & & 167.6, C & & $169.8, \mathrm{C}$ \\
\hline 2 & $8.40, \mathrm{~s}$ & & $8.68, \mathrm{~s}$ & \\
\hline 3 & $4.68, \mathrm{~d}(1.8)$ & $58.0, \mathrm{CH}$ & & $84.9, \mathrm{C}$ \\
\hline $3-\mathrm{OH}$ & & & $6.58, \mathrm{~s}$ & \\
\hline 4 & & 147.3, C & & 150.1, C \\
\hline 5 & & & & \\
\hline 6 & & 131.1, C & & 141.1, C \\
\hline 7 & & 154.1, C & $7.64, \mathrm{~d}(8.8)$ & $129.6, \mathrm{CH}$ \\
\hline 7-OH & $9.80, \mathrm{~s}$ & & & \\
\hline 8 & $6.83, \mathrm{~d}(2.7)$ & 107.7, CH & $7.46, \mathrm{~d}(8.8)$ & 125.0, CH \\
\hline 9 & & 158.6, C & & 158.7, C \\
\hline 10 & $6.99, \mathrm{~d}(2.7)$ & 97.0, $\mathrm{CH}$ & & $106.8, \mathrm{C}$ \\
\hline 11 & & $120.9, \mathrm{C}$ & & 121.1, C \\
\hline 12 & & $160.2, \mathrm{C}$ & & 161.1, C \\
\hline 13 & & & & \\
\hline 14 & $4.91, \mathrm{~d}(8.7)$ & $60.4, \mathrm{CH}$ & $4.90, \mathrm{~d}(8.8)$ & $60.7, \mathrm{CH}$ \\
\hline 15 & $2.24, \mathrm{~m}$ & $30.4, \mathrm{CH}$ & $2.62, \mathrm{~m}$ & $32.9, \mathrm{CH}$ \\
\hline 16 & $0.83, \mathrm{~d}(6.7)$ & $19.8, \mathrm{CH}_{3}$ & $1.10, \mathrm{~d}(6.4)$ & 20.7, $\mathrm{CH}_{3}$ \\
\hline 17 & $1.03, \mathrm{~d}(6.7)$ & $19.1, \mathrm{CH}_{3}$ & $0.79, \mathrm{~d}(6.4)$ & $19.7, \mathrm{CH}_{3}$ \\
\hline 18 & $2.93, \mathrm{~m}$ & $34.7, \mathrm{CH}$ & $2.71, \mathrm{~m}$ & 41.6, CH \\
\hline 19 & $1.13, \mathrm{~d}(7.3)$ & $15.4, \mathrm{CH}_{3}$ & $1.13, \mathrm{~d}(6.4)$ & $11.4, \mathrm{CH}_{3}$ \\
\hline 20 & $1.24, \mathrm{~m}$ & 22.7, $\mathrm{CH}_{2}$ & $1.12, \mathrm{~m} 1.20, \mathrm{~m}$ & 25.0, $\mathrm{CH}_{2}$ \\
\hline 21 & $0.83, \mathrm{t}(7.2)$ & $12.4, \mathrm{CH}_{3}$ & $0.84, \mathrm{t}(7.1)$ & $13.1, \mathrm{CH}_{3}$ \\
\hline 22 & $3.82, \mathrm{~s}$ & $55.5, \mathrm{CH}_{3}$ & $3.89, \mathrm{~s}$ & $56.2, \mathrm{CH}_{3}$ \\
\hline
\end{tabular}

${ }^{\text {a }}$ Measured at $600 \mathrm{MHz}$

${ }^{\mathrm{b}}$ Measured at $150 \mathrm{MHz}$

${ }^{\mathrm{c}}$ Measured at $400 \mathrm{MHz}$

${ }^{\mathrm{d}}$ Measured at $100 \mathrm{MHz}$

\subsection{Spectroscopic Data of Compounds}

\subsubsection{Versicomide $E$ (1)}

Faint yellow powder; $[\alpha]_{\mathrm{D}}^{20}=-88.5(c 0.5, \mathrm{MeOH})$; UV $(\mathrm{MeOH}) \lambda_{\max }(\log \varepsilon) 209$ (2.66), $242(3.11) \mathrm{nm}$; IR (KBr) $\nu_{\max } 1662,1629,1590,1467,1383 \mathrm{~cm}^{-1}$; ECD (MeOH): $200(\Delta \varepsilon+3.49), 229(\Delta \varepsilon-5.13) \mathrm{nm} ;{ }^{1} \mathrm{H}$ and ${ }^{13} \mathrm{C}$ NMR data: Table 1; HRESIMS $m / z 360.1919$ for $[\mathrm{M}+\mathrm{H}]^{+}$(calcd 360.1923 for $\mathrm{C}_{19} \mathrm{H}_{25} \mathrm{~N}_{3} \mathrm{O}_{4}$ ).

\subsubsection{Versicomide F (2)}

Faint yellow jelly; $[\alpha]_{\mathrm{D}}^{20}=-32.4(c 1.0, \mathrm{MeOH})$; UV $(\mathrm{MeOH}) \lambda_{\max }(\log \varepsilon) 226$ (2.62), $279(0.95) \mathrm{nm}$; IR (KBr) $\nu_{\max } 1677,1617,1597,1492,1361 \mathrm{~cm}^{-1}$; ECD (MeOH): 219 $(\Delta \varepsilon+2.59), 238(\Delta \varepsilon-4.83) \mathrm{nm} ;{ }^{1} \mathrm{H}$ NMR and ${ }^{13} \mathrm{C}$ NMR data: Table 1; HRESIMS $m / z 360.1919$ for $[\mathrm{M}+\mathrm{H}]^{+}$(calcd 360.1923 for $\mathrm{C}_{19} \mathrm{H}_{25} \mathrm{~N}_{3} \mathrm{O}_{4}$ ).

\subsection{Anti-inflammatory Activity Test}

The inhibition of NO production assay was performed according to the reported procedures [26]. DiDOX was set up as a positive control group. Then the percentage of NO production inhibition was calculated to get the $\mathrm{IC}_{50}$ values.

Acknowledgements The authors gratefully acknowledge the National Natural Science Foundation of China (Nos. 81874293 and 81630093) for financial support and Major Basic Research Program of Shandong Province (ZR2019ZD26).

\section{Compliance with Ethical Standards}

Conflict of interest The authors declare no conflict of interest.

Open Access This article is licensed under a Creative Commons Attribution 4.0 International License, which permits use, sharing, adaptation, distribution and reproduction in any medium or format, as long as you give appropriate credit to the original author(s) and the source, provide a link to the Creative Commons licence, and indicate if changes were made. The images or other third party material in this article are included in the article's Creative Commons licence, unless indicated otherwise in a credit line to the material. If material is not included in the article's Creative Commons licence and your intended use is not permitted by statutory regulation or exceeds the permitted use, you will need to obtain permission directly from the copyright holder. To view a copy of this licence, visit http://creativecommons.org/licenses/by/4.0/.

\section{References}

1. R.N. Kharwar, A. Mishra, S.K. Gond, A. Stierle, D. Stierle, Nat. Prod. Rep. 28, 1208-1228 (2011)

2. M. Rajamanikyam, V. Vadlapudi, R. Amanchy, S.M. Upadhyayula, Braz. Arch. Biol. Technol. 60, e17160542, ISSN 1678-4324 (2017)

3. E.O. Omeje, J.E. Ahomafor, T.U. Onyekaba, P.O. Monioro, I. Nneka, S. Onyeloni, C. Chime, J.C. Eboka, Natural Products and Cancer Drug Discovery (InTech, London, 2017).

4. S.X. Cai, S.W. Sun, H.N. Zhou, X.L. Kong, T.J. Zhu, D.H. Li, Q.Q. Gu, J. Nat. Prod. 74, 1106-1110 (2011)

5. G. Ding, Y. Li, S.B. Fu, S.C. Liu, J.C. Wei, Y.S. Che, J. Nat. Prod. 72, $182(2009)$

6. W. Wu, H.Q. Dai, L. Bao, B. Ren, J.C. Lu, Y.M. Luo, L.D. Guo, L.X. Zhang, H.W. Liu, J. Nat. Prod. 74, 1303 (2011)

7. K. Ma, J.J. Han, L. Bao, T.Z. Wei, H.W. Liu, J. Nat. Prod. 77, 942-947 (2014)

8. Z.K. Guo, T. Yan, Y. Guo, Y.C. Song, R.H. Jiao, R.X. Tan, H.M. Ge, J. Nat. Prod. 75, 15-21 (2012)

9. T. El-Elimat, M. Figueroa, H.A. Raja, T.N. Graf, A.F. Adcock, D.J. Kroll, C.S. Day, M.C. Wani, C.J. Pearce, N.H. Oberlies, J. Nat. Prod. 76, 382-387 (2013)

10. M.I. Bidartondo, J.G. Duckett, Proc. R. Soc. B 277, 485-492 (2010) 
11. W. Peng, L. Guo, C.J. Zheng, Biochem. Syst. Ecol. 45, 124-126 (2012)

12. X.N. Wang, B.P. Bashyal, E.M.K. Wijeratne, J. Nat. Prod. 74, 2052-2061 (2011)

13. X.B. Li, F. Xie, S.S. Liu, Chem. Biodivers. 10, 1193-1201 (2013)

14. X.Q. Song, X. Zhang, Q.J. Han, Phytochem. Lett. 6, 318-321 (2013)

15. Y. Jiao, X. Zhang, L. Wang, Phytochem. Lett. 6, 14-17 (2013)

16. C.Q. Pan, Y.T. Shi, X.G. Chen, C.-T.A. Chen, X.Y. Tao, B. Wu, Org. Biomol. Chem. 15, 1155-1163 (2017)

17. J.F. Wang, W.J. He, X.L. Huang, X.P. Tian, S.R. Liao, B. Yang, F.Z. Wang, X.J. Zhou, Y.H. Liu, J. Agric. Food Chem. 64, 29102916 (2016)

18. T.J. Greshock, A.W. Grubbs, P. Jiao, D.T. Wicklow, J.B. Gloer, R.M. Williams, Angew. Chem. Int. Ed. 60, 3573-3577 (2008)

19. I. Kagiyama, H. Kato, T. Nehira, J.C. Frisvad, D.H. Sherman, R.M. Williams, S. Tsukamoto, Angew. Chem. Int. Ed. 55, 11281132 (2016)
20. H. Kato, T. Yoshida, T. Tokue, Y. Nojiri, H. Hirota, T. Ohta, R.M. Williams, S. Tsukamoto, Angew. Chem. Int. Ed. 46, 2254-2256 (2007)

21. B. Wang, E.M. Park, J.B. King, A.O. Mattes, S.L. Nimmo, C. Clendinen, A.S. Edison, C. Anklin, R.H. Cichewicz, J. Nat. Prod. 78, 1415-1421 (2015)

22. Z.Z. Zhao, K.Y. Han, Z.H. Li, T. Feng, H.P. Chen, J.K. Liu, Phytochem. Lett. 30, 143-149 (2019)

23. W. Ebrahim, M. El-Neketi, L.I. Lewald, R.S. Orfali, W. Lin, N. Rehberg, R. Kalscheuer, G. Daletos, P. Proksch, J. Nat. Prod. 79, 914-922 (2016)

24. Y.Q. Tian, X.C. Qin, X.P. Lin, K. Kaliyaperumal, X.F. Zhou, J. Liu, Z. Ju, Z.C. Tu, Y.H. Liu, J. Antibiot. 68, 703-706 (2015)

25. Z. Wu, Y.R. Wang, D. Liu, P. Proksch, S.W. Yu, W.H. Lin, Tetrahedron (2015). https://doi.org/10.1016/j.tet.2015.10.038

26. Y.R. Li, G.H. Li, L. Sun, L. Li, Y. Liu, D.G. Kong, S.Q. Wang, D.M. Ren, X.N. Wang, H.X. Lou, T. Shen, Oxid. Med. Cell. Longev. (2018). https://doi.org/10.1155/2018/7616852 